\title{
Pseudo-Goldstone dark matter confronts cosmic ray and collider anomalies
}

\author{
James M. Cline and Takashi Toma \\ McGill University, Department of Physics, 3600 University Street, Montréal, Quebec H3A2T8, Canada
}

(Received 11 June 2019; published 26 August 2019)

\begin{abstract}
Persistent excesses in the spectra of gamma rays from the Galactic Center and cosmic ray antiprotons can be explained by dark matter of mass $50-65 \mathrm{GeV}$ annihilating into $b$ quarks, but this is typically hard to reconcile with direct detection constraints. We resolve this tension using a simple class of models, where dark matter is a pseudo-Nambu-Goldstone boson, having naturally momentum-suppressed couplings to nuclei. Exploring the parameter space of the model, we find that it can explain the cosmic ray anomalies while remaining compatible with constraints from the relic abundance and annihilation in dwarf spheroidal galaxies. In certain regions of parameter space, the Higgs-dark matter coupling can help stabilize the Higgs potential up to the Planck scale. The scalar partner of the dark matter is an extra Higgs boson, that can explain a tentative diphoton excess observed by CMS, and an excess of $b \bar{b}$ signal from LEP, if its mass is $\sim 96 \mathrm{GeV}$ and the model is extended to include a heavy scalar quark. This extended model predicts a monochromatic gamma-ray line near $64 \mathrm{GeV}$, at a level close to current experimental sensitivity, from dark matter annihilations in the Galaxy.
\end{abstract}

DOI: 10.1103/PhysRevD.100.035023

\section{INTRODUCTION}

Although there is so far no clear evidence for nongravitational interactions of dark matter (DM), it has been suggested that excess of $\mathrm{GeV}$-scale gamma rays from the Galactic Center (GC), observed by the Fermi-LAT [1,2], could be due to DM annihilations [3-9]. A leading alternative astrophysical explanation is that the excess comes from an unresolved population of millisecond pulsars or other astrophysical sources [10-14], although arguments against the pulsar hypothesis have been presented [15-17]. Until such possible point sources are resolved, the DM hypothesis remains viable. In particular, Ref. [18] has recently called into question claims that the statistical properties of the $\mathrm{GeV}$ excess are more consistent with unresolved point sources than with DM annihilations $[19,20]$, rekindling the motivation to consider particle physics models for the GC excess.

At the same time, the AMS Collaboration obtained the first precise measurement of the spectrum of cosmic ray antiprotons [21]. It has been analyzed by numerous groups who find an excess over the expected flux at energies of $10-20 \mathrm{GeV}$ [22-28]. Intriguingly, this signal can be simultaneously explained with the GC gamma-ray excess by DM annihilating into $b \bar{b}$ [29-31]. In this paper we

Published by the American Physical Society under the terms of the Creative Commons Attribution 4.0 International license. Further distribution of this work must maintain attribution to the author(s) and the published article's title, journal citation, and DOI. Funded by SCOAP . propose that both the $\mathrm{GC}$ and the $\bar{p}$ excesses can be explained by the annihilation of pseudo-Nambu-Goldstone dark matter (pNGB DM).

A natural explanation for why DM $(\chi)$ should annihilate mainly into $b \bar{b}$ is the Higgs portal, since $h \rightarrow b \bar{b}$ is the dominant decay channel of the Higgs boson. However this tends to be strongly ruled out by direct detection constraints [32], because $h$ couples relatively strongly to nucleons [33]. For this reason, singlet scalar DM in the mass range of interest is excluded except for $m_{\chi} \in(55-62.5) \mathrm{GeV}$, just below the Higgs resonance [34,35]. But for $m_{\chi}<m_{h} / 2$, the cross section for $\chi \chi \rightarrow b \bar{b}$ at present times is highly suppressed compared to that during the time of freeze-out in the early Universe (as we will discuss below), making singlet scalar DM unsuitable for explaining the cosmic ray excesses.

One way of circumventing the direct detection constraints for Higgs portal models is by taking the DM to be fermionic, coupling to $b$ quarks through a pseudoscalar such that DM-nucleon interactions are spin dependent and velocity suppressed [36-40]. Here we will use a different strategy, taking advantage of an approximate symmetry, following Refs. [41-43]. There a simple class of models was considered where the required cancellation for direct detection occurs as consequence of a softly broken global $\mathrm{U}(1)$ symmetry, rather than through fine-tuning. The DM candidate is the pseudo-Nambu-Goldstone boson arising from spontaneous breaking of the global U(1) symmetry, with Higgs portal couplings.

In this case, the pNGB retains the feature of being derivatively coupled with other particles. Its interaction 
with matter is therefore highly suppressed for nonrelativistic DM, as in direct detection experiments. Loop corrections do not have this feature because of the explicit breaking of the global U(1) symmetry. This has been studied in Refs. [44,45], where it was found that loop corrections are nevertheless very small and well within current direct detection bounds.

The pNGB dark matter model is quite economical, with only four free parameters: the DM mass $m_{\chi}$, the mass of its scalar partner (a second Higgs boson $h_{2}$ ), the mixing angle $\theta$ between $h_{2}$ and the observed Higgs $h_{1}$, and the vacuum expectation value (VEV) $v_{s}$ that spontaneously breaks the $\mathrm{U}(1)$ symmetry (or alternatively the quartic coupling $\lambda_{S}$ of the new complex scalar field $S$ ). In Ref. [43], it was shown that one can easily satisfy the requirements of getting the correct relic density, while respecting other constraints like the Higgs invisible width and perturbative unitarity. Moreover, probing the pNGB DM at the LHC has been discussed in Ref. [46], and further generalizations of the model have been considered $[47,48]$. However its implications for indirect detection have not been considered prior to this work.

A possible bonus of the pNGB DM model is that it can enhance the stability of the Higgs quartic coupling to higher renormalization scales, in contrast to the SM prediction that the Higgs quartic coupling should become negative around $10^{11} \mathrm{GeV}$ [49], triggering an instability. This is a consequence of the Higgs portal coupling, that affects the renormalization group evolution of the Higgs quartic coupling [50-54]. We identify regions of parameter space where the instability can be avoided and the quartic couplings are perturbative up to the Planck scale, while satisfying the relic abundance constraints and accounting for the cosmic ray excesses.

A further set of tentative anomalies has been identified in results coming from the LEP and CMS experiments, that both point to a new Higgs-like particle $h_{2}$ at a mass close to $96 \mathrm{GeV}$. The LEP anomaly is a mild excess $b \bar{b}$ pairs that would arise from decays of $h_{2}$ following $e^{+} e^{-} \rightarrow Z h_{2}$ production via Higgs-strahlung [55]. At a similar mass, CMS observes a $2.9 \sigma$ local excess in the diphoton channel, that could come from $g g \rightarrow h_{2} \rightarrow \gamma \gamma$ [56-58]. These observations have motivated theorists to propose a number of models [59-68]. A necessary ingredient of the pNGB DM model is an additional scalar $h_{2}$ that mixes with the SM Higgs, denoted as $h_{1}$. It is possible to take $m_{h_{2}}=96 \mathrm{GeV}$ while still being consistent with the cosmic ray excesses. Although the LEP observation could come directly from the pNGB DM setup, given large enough mixing between the two scalars, the CMS excess requires an additional charged and colored scalar $\Phi$ coupling to the singlet. We make a systematic study of the potential for such extensions of the model to simultaneously explain both tentative signals as well as the collider anomalies. We show that the extended model including the colored scalar provides an additional means of discovering pNGB DM, by its annihilation to monochromatic gamma rays in the Galaxy.

The paper is organized as follows. We review the framework of pNGB DM in Sec. II and then show in Sec. III that it can account for the anomalous cosmic ray signals in gamma rays and antiprotons, for DM mass near $m_{\chi} \sim 65 \mathrm{GeV}$, over wide ranges of the other free parameters. In Sec. IV we explore Higgs stability and perturbativity, showing that it can be achieved if $m_{h_{2}} \gtrsim 140 \mathrm{GeV}$. Section V analyzes a broad class of models that can potentially explain the LEP and CMS anomalies, singling out one that is most promising. We summarize and give conclusions in Sec. VI.

\section{PNGB DARK MATTER}

We begin by reviewing the particle physics model, which has just one additional complex scalar field $S=$ $\left(v_{s}+s+i \chi\right) / \sqrt{2}$ relative to the standard model (SM). The scalar potential is given by

$$
\begin{aligned}
\mathcal{V}= & -\frac{\mu_{H}^{2}}{2}|H|^{2}+\frac{\lambda_{H}}{2}|H|^{4}-\frac{\mu_{S}^{2}}{2}|S|^{2}+\frac{\lambda_{S}}{2}|S|^{4} \\
& +\lambda_{H S}|H|^{2}|S|^{2}-\frac{m_{\chi}^{2}}{4}\left(S^{2}+S^{* 2}\right),
\end{aligned}
$$

where the last term explicitly breaks the global U(1) symmetry $S \rightarrow e^{i \alpha} S$, giving mass $m_{\chi}$ to the NGB DM candidate $\chi$. This explicit breaking term can be derived from an extended model with gauged U(1) symmetry at high energy scale [43]. From the viewpoint of technical naturalness, there is no need for the soft-breaking mass to be small compared to $\mu_{S}^{2}$; moreover the model's mechanism for suppressing direct detection signals does not require any hierarchy between the two mass scales.

With no loss of generality, $m_{\chi}^{2}$ can be made real valued by absorbing its phase with a field redefinition of $S$. Then the DM $\chi$ remains stable due to a $\mathbb{Z}_{2}$ symmetry even after $S$ gets its VEV. The field $H$ is the SM Higgs doublet, $H=(0,(v+h) / \sqrt{2})^{T}$. The VEVs $v_{s}$ and $v$ are

$$
\left(\begin{array}{c}
v^{2} \\
v_{s}^{2}
\end{array}\right)=\frac{1}{\lambda_{H} \lambda_{S}-\lambda_{H S}^{2}}\left(\begin{array}{cc}
\lambda_{S} & -\lambda_{H S} \\
-\lambda_{H S} & \lambda_{H}
\end{array}\right)\left(\begin{array}{c}
\mu_{H}^{2} \\
\mu_{S}^{2}+m_{\chi}^{2}
\end{array}\right) .
$$

The mass matrix for $h$ and $s$ can be written as

$$
M^{2}=\left(\begin{array}{cc}
\lambda_{H} v^{2} & \lambda_{H S} v v_{s} \\
\lambda_{H S} v v_{s} & \lambda_{S} v_{s}^{2}
\end{array}\right)
$$

which is diagonalized by the rotation

$$
\left(\begin{array}{l}
h \\
s
\end{array}\right)=\left(\begin{array}{cc}
c_{\theta} & s_{\theta} \\
-s_{\theta} & c_{\theta}
\end{array}\right)\left(\begin{array}{l}
h_{1} \\
h_{2}
\end{array}\right),
$$


with $c_{\theta}=\cos \theta, s_{\theta}=\sin \theta$, and the mixing angle given by

$$
\tan 2 \theta=\frac{2 \lambda_{H S} v v_{s}}{\lambda_{S} v_{s}^{2}-\lambda_{H} v^{2}}
$$

The mass eigenstate $h_{1}$ is identified as the SM-like Higgs boson with mass $m_{h_{1}}=125 \mathrm{GeV}$, and $h_{2}$ is a new $C P$-even state. The trilinear couplings of the DM to the $h_{i}$ bosons are expressed in terms of $\theta$ and the masses as

$$
\mathcal{V} \supset-\frac{s_{\theta} m_{h_{1}}^{2}}{2 v_{s}} h_{1} \chi^{2}+\frac{c_{\theta} m_{h_{2}}^{2}}{2 v_{s}} h_{2} \chi^{2}
$$

while the $h_{i}$ couplings to SM fermions $f$ have the form

$$
\mathcal{L} \supset-\frac{m_{f}}{v}\left(c_{\theta} h_{1}+s_{\theta} h_{2}\right) \bar{f} f .
$$

From the structure of Eqs. (6) and (7), one can see that the matrix element for $\chi f \rightarrow \chi f$ scattering mediated by $h_{1,2}$ exchange exactly vanishes at zero momentum transfer, which is the key feature for making the model safe from direct detection constraints. On the other hand for the annihilation $\chi \chi \rightarrow f \bar{f}$, the cancellation is ineffective as long as $m_{h_{1}} \approx m_{h_{2}}$, since the $s$-channel propagator carries significant momentum. The main annihilation channel determining the DM relic abundance is $\chi \chi \rightarrow b \bar{b}$ for $5 \mathrm{GeV} \lesssim m_{\chi} \lesssim 70 \mathrm{GeV}$, covering the mass range that is relevant for the present work. ${ }^{1}$ The DM annihilation cross section for $\chi \chi \rightarrow b \bar{b}$ is given by

$$
\begin{aligned}
\sigma_{b \bar{b}} v_{\text {rel }} \approx & \frac{c_{\theta}^{2} s_{\theta}^{2} m_{b}^{2}}{4 \pi v^{2} v_{s}^{2}}\left(1-4 \frac{m_{b}^{2}}{m_{\chi}^{2}}\right)^{3 / 2} \\
& \times\left|\frac{m_{h_{1}}^{2}}{s-m_{h_{1}}^{2}+i m_{h_{1}} \Gamma_{h_{1}}}-\frac{m_{h_{2}}^{2}}{s-m_{h_{2}}^{2}+i m_{h_{2}} \Gamma_{h_{2}}}\right|^{2},
\end{aligned}
$$

where $\sqrt{s}$ is the total DM energy in the center of mass frame, and $\Gamma_{h_{i}}$ is the total decay width of $h_{i}$. For quantitative determination of the DM relic abundance, we use the full thermally averaged annihilation cross section $\left\langle\sigma v_{\text {rel }}\right\rangle$ computed using MicrOmegas [69].

On the other hand for annihilation within our Galaxy, Eq. (8) at $s=4 m_{\chi}^{2}$ provides a very good approximation in the DM mass region we consider because the DM is very slowly moving, $v \sim 10^{-3} c$, and its kinetic energy is much smaller than the Higgs decay width, $\left(s-4 m_{\chi}^{2}\right) / m_{\chi} \sim$ $m_{\chi} v^{2} \ll \Gamma_{h_{i}}$. Therefore the phase space averaging has a negligible effect on Eq. (8) even exactly on the resonance

\footnotetext{
${ }^{1}$ See the next section for discussion of the subdominant $\chi \chi \rightarrow W W$ annihilation channel.
}

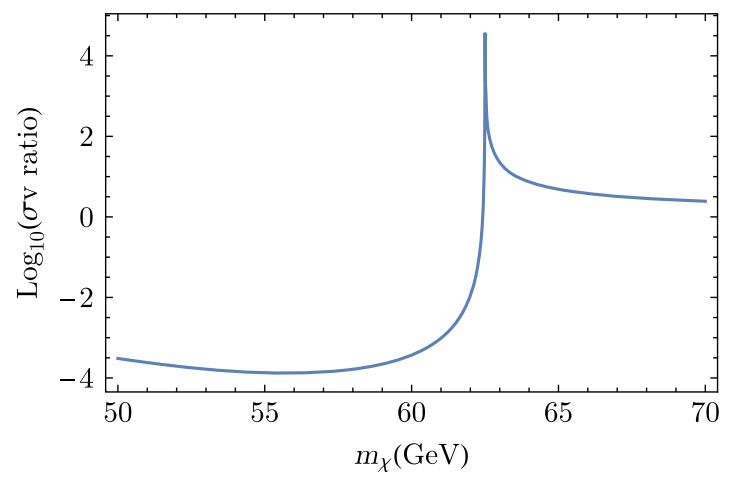

FIG. 1. Ratio of $\left\langle\sigma_{b \bar{b}} v_{\text {rel }}\right\rangle$ at present times to that in the early Universe, taking the freeze-out temperature to be $T=m_{\chi} / 20$.

$4 m_{\chi}^{2}=m_{h_{i}}^{2}$, and we can use this expression for predicting indirect detection signals.

It is interesting that Eq. (8) can differ significantly from $\left\langle\sigma_{b \bar{b}} v_{\text {rel }}\right\rangle$ in the early Universe near the resonance at $m_{\chi}=$ $m_{h_{i}} / 2$ [70]. We illustrate this in Fig. 1 by plotting the ratio of $\left\langle\sigma_{b \bar{b}} v_{\text {rel }}\right\rangle$ at late times versus at the time of freeze-out as a function of $m_{\chi}$. This shows that the indirect signal is suppressed by orders of magnitude for $m_{\chi}<m_{h} / 2$ and motivates our search for models with $m_{\chi}>m_{h} / 2$, where the indirect signal is moderately enhanced, but can nevertheless satisfy Fermi dwarf spheroidal constraints [71-73].

If $m_{\chi}<m_{h_{1}} / 2 \approx 62.5 \mathrm{GeV}$, the trilinear coupling in Eq. (6) gives rise to an invisible decay channel for the Higgs boson, $h_{1} \rightarrow \chi \chi$, whose partial width is

$$
\Gamma_{\mathrm{inv}}=\frac{s_{\theta}^{2} m_{h_{1}}^{3}}{32 \pi v_{s}^{2}} \sqrt{1-4 \frac{m_{\chi}^{2}}{m_{h_{1}}^{2}}} .
$$

The branching ratio for invisible decays is experimentally constrained at the level of $\mathrm{BR}_{\mathrm{inv}}<0.19$ by the CMS Collaboration [74], and 0.26 by the ATLAS Collaboration [75]. Ignoring the phase space suppression (which is valid for $m_{\chi} \lesssim 60 \mathrm{GeV}$ ), this gives the approximate constraint $s_{\theta} / v_{s} \lesssim 0.2 \mathrm{TeV}^{-1}$.

\section{FITTING COSMIC RAY EXCESSES}

We performed a numerical scan of the parameter space, to look for regions compatible with the gamma-ray and antiproton excesses, as well as DM relic abundance and dwarf spheroidal constraints on DM annihilation. The results are shown in Fig. 2, where the extra Higgs boson mass takes the values $m_{h_{2}}=70,96,300 \mathrm{GeV}$ in successive rows.

In the left panels, the purple lines show the relation between $\lambda_{S}$ and $m_{\chi}$ that gives the correct DM relic abundance, for several values of the mixing angle, $s_{\theta}=$ 0.01 (solid), 0.10 (dashed), 0.30 (dot-dashed). The upper gray and blue regions are excluded by the perturbative unitarity bound $\lambda_{S}<8 \pi / 3$ [78], and the Higgs invisible 

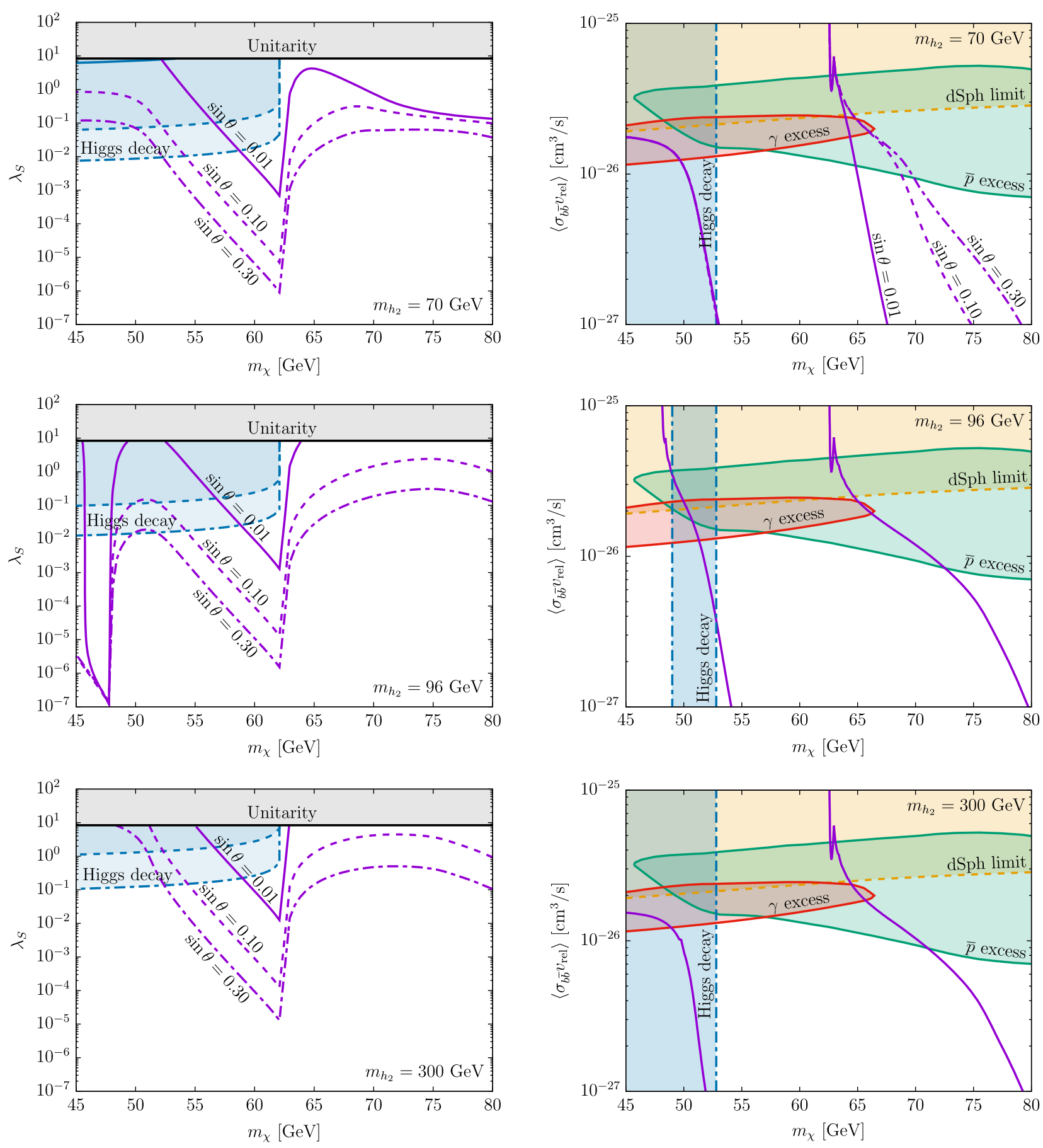

FIG. 2. Left: Constraints from the thermal relic abundance (purple curves), Higgs invisible width (blue region), and perturbative unitarity (gray region, $\lambda_{S}>8 \pi / 3$ ). The mixing angle $\sin \theta$ is varied between 0.01 (solid), 0.1 (dashed) and 0.3 (dot-dashed). The new $C P$-even Higgs mass is $m_{h_{2}}=70 \mathrm{GeV}$ (top), $96 \mathrm{GeV}$ (middle) and $300 \mathrm{GeV}$ (bottom). Right: Predicted annihilation cross section for $\chi \chi \rightarrow b \bar{b}$ at late times, relevant annihilation in the Milky Way, versus $m_{\chi}$. Purple curves are fixed by the relic density, while the red and green shading denote the $2 \sigma$ allowed regions [29] for gamma-ray and antiproton excesses, respectively. The blue and orange regions are excluded by the Higgs invisible width and gamma-ray observations from dSphs [76,77], respectively.

decay constraint $\mathrm{BR}_{\mathrm{inv}}<0.19$ [74], respectively. We remark that the relic density curves may be affected by early kinetic decoupling of the DM if its mass is just below the Higgs resonance $\left(m_{\chi} \lesssim m_{h} / 2\right)[79,80]$. As a result, the Higgs invisible decay constraint may change by a factor $\lesssim 7$ [81]. However this does not impact the region of parameter space relevant for explaining the cosmic ray anomalies, so we do not take account of this effect. 
In the right panels of Fig. 2, the relic abundance curves (purple) are replotted in the plane of the DM mass and the $s$-wave annihilation cross section, evaluated at the present time, which is relevant for indirect detection. Along each curve, the quartic coupling $\lambda_{S}$ (or equivalently $v_{s}$ ) is fixed to give the correct relic abundance. To fit the cosmic ray excesses, we adopt the $2 \sigma$ allowed regions found in Ref. [29], plotted as the red and green regions for the gamma ray and antiproton signals, respectively. For each of the three chosen $m_{h_{2}}$ values, there is some overlap between all the allowed regions, roughly for DM masses in the range $64 \mathrm{GeV} \lesssim m_{\chi} \lesssim 67 \mathrm{GeV}$. The regions excluded by invisible Higgs decays can be inferred from the corresponding left-hand graph by noticing where the relic density curve goes above the Higgs decay curve for a given mixing angle. It happens that these excluded regions nearly coincide in mass, independently of $\theta$, leading to only a single excluded region in the right-hand plots.

For all but the lowest value of $m_{h_{2}}$, the allowed regions are independent of the mixing angle $\theta$, because only the combination $s_{\theta} / v_{s}$ appears in the $b \bar{b}$ annihilation cross section given by Eq. (8). For $m_{h_{2}}=70 \mathrm{GeV}$ however, the additional annihilation channel $\chi \chi \rightarrow h_{2} h_{2}$ becomes important, breaking this degeneracy. We emphasize that in generic models such as singlet scalar DM, the allowed region for explaining both excesses is excluded by the strong direct detection bound, or suppression of the indirect signal.

The allowed regions are also compatible with the most recent constraints from DM annihilation in satellite galaxies of the Milky Way [76,77], shown in orange. This exclusion is based on gamma-ray observations of 28 confirmed dwarf spheroidal galaxies (dSph) and 17 new candidate systems, taking advantage of spectroscopically determined $J$ factors for the known dSphs and and predicted $J$ factors for the candidates. It gives a limit that is 1.5 times weaker than in previous determinations.

In our numerical analysis, only the annihilation channel $\chi \chi \rightarrow b \bar{b}$ is taken into account for the gamma-ray and antiproton excesses. However for heavy DM, the annihilation into $W W^{*}$ may dominate. We have checked that for $m_{\chi}$ in the range $55 \mathrm{GeV} \lesssim m_{\chi} \lesssim 65 \mathrm{GeV}$, the branching fraction into $W W^{*}$ can be $25 \%$ at most, and the ensuing shapes of the energy spectra of gamma rays and antiprotons are only slightly modified as a result.

\section{POTENTIAL STABILITY AND PERTURBATIVITY}

Another interesting feature of our model is that the allowed values of the Higgs portal coupling $\lambda_{H S}$ can be compatible with extending the stability of the Higgs potential up to the Planck scale [50-54]. It is well established that the Higgs quartic coupling in the SM runs to negative values at high energy scales $\Lambda_{I} \sim 10^{11} \mathrm{GeV}$ under renormalization [49]. This could lead to a catastrophic destabilization of the electroweak vacuum in the early Universe, if inflation occurred at a sufficiently high scale [82].

There are two ways in which the Higgs portal coupling $\lambda_{H S}$ can improve stability. First, it provides an additional positive contribution to the $\beta$ function of $\lambda_{H}$. Second, $\lambda_{H}$ gets a positive threshold correction at the scale $\mu=m_{h_{2}}$ when $h_{2}$ is integrated out. The running $\lambda_{H}$ is increased by $\delta \lambda_{H} \equiv$ $\lambda_{H S}^{2} / \lambda_{S}$ as the scale $\mu$ crosses the threshold from below. For $\lambda_{H S}>0$, the stability condition also becomes more stringent at this scale, $\lambda_{H}>\delta \lambda$ instead of $\lambda_{H}>0$. But as the scale $\mu$ becomes sufficiently large compared to $m_{h_{2}}$, the condition relaxes to the naive requirement $\lambda_{H}(\mu)>0$, so that the threshold correction can overcome the tendency for $\lambda_{H}\left(\Lambda_{I}\right)$ to become negative. The $\beta$ functions of the couplings at twoloop level are obtained using SARAH [83,84]. Here we concentrate on the $\lambda_{H S}>0$ case since this has been shown to be more effective for Higgs stability than $\lambda_{H S}<0$ [51].

In Fig. 3 we display the regions $\sin \theta-\lambda_{S}$ plane that correspond to models with Higgs stability up to the Planck scale (purple region) and that can explain the cosmic ray excesses (red region). The upper plot illustrates the situation for $m_{h_{2}}=300 \mathrm{GeV}$. The regions above and to the right of the curves labeled by $\Lambda=10^{5}, 10^{10}, 10^{20} \mathrm{GeV}$ have a Landau pole in one of the quartic couplings $\lambda_{H}, \lambda_{S}$ and $\lambda_{H S}$ at the scale indicated. Thus a fully UV-complete theory can be achieved for parameters in the range $0.1 \lesssim$ $\sin \theta \lesssim 0.3$ and $0.06 \lesssim \lambda_{S} \lesssim 0.4$. The threshold correction, rather than the extra contribution of $\lambda_{H S}$ to the $\beta$ function, is the most important effect for achieving stability in the overlap region. For $\sin \theta \gtrsim 0.4$, it gives too large an enhancement in $\lambda_{H}$, causing it to blow up at scales $\Lambda$ below the Planck scale; this is the reason the Landau pole curves are vertical at large $\sin \theta$.

In the lower plot of Fig. 3, we show the allowed parameters corresponding to higher values of $m_{h_{2}}$. Here the purple shaded regions combine the requirements of stability of the Higgs potential and perturbativity of the quartic couplings, considering $m_{h_{2}}=300,2000$ and $10^{4} \mathrm{GeV}$, requiring smaller $\sin \theta$ with increasing $m_{h_{2}}$. The allowed region for the cosmic ray anomalies shrinks rapidly with increasing $m_{h_{2}}$. For $m_{h_{2}} \gtrsim 600 \mathrm{GeV}$, the overlap of the stability and anomaly regions disappears. Moreover for $m_{h_{2}} \lesssim 140 \mathrm{GeV}$, there is no longer any region of stability/ perturbativity [53].

\section{LEP AND CMS ANOMALIES}

There are experimental hints for an additional Higgs-like state with mass near $96 \mathrm{GeV}$. In their search for the SM Higgs, the combined LEP Collaborations observed a $2.3 \sigma$ excess of $b \bar{b}$ final states at $m \sim 98 \mathrm{GeV}$, corresponding to a signal strength approximately 10 times lower than would be produced by a SM Higgs of this mass [55].

More recently, in a search for the diphoton final state, CMS reported a $2.9 \sigma$ (local significance) excess in the 

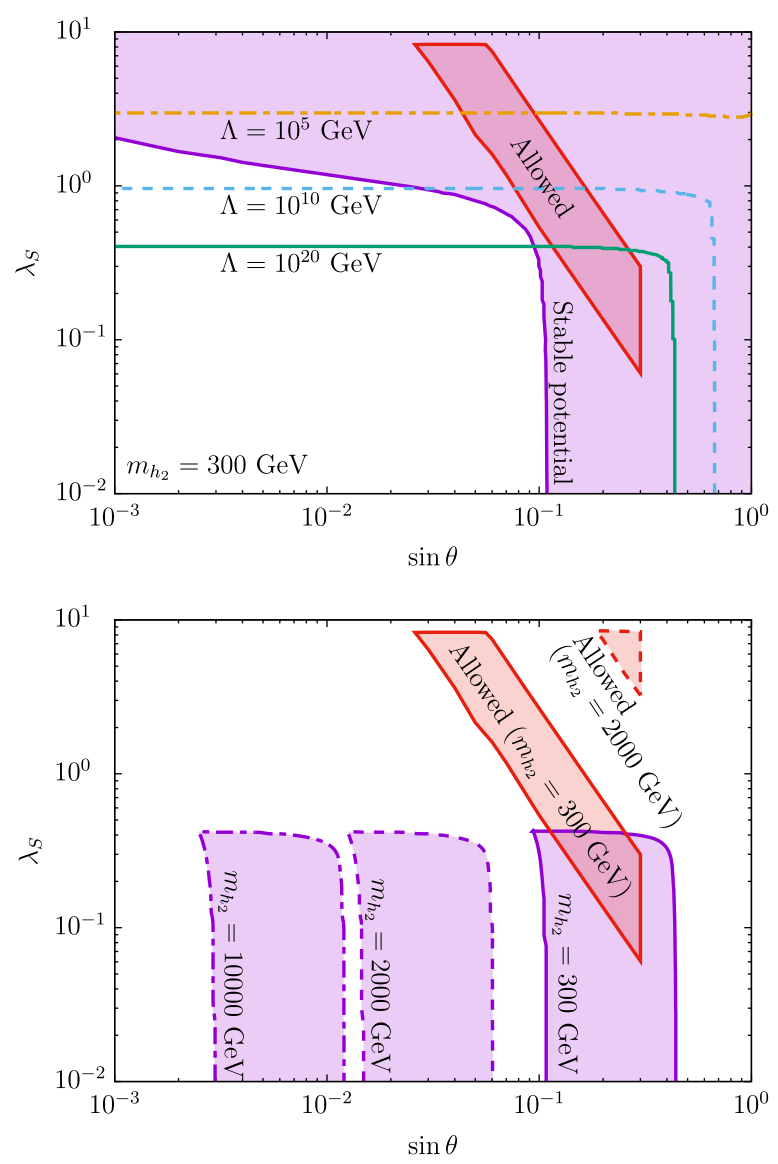

FIG. 3. Top: Regions $\lambda_{S}$ versus $\sin \theta$ where the scalar potential is stable and perturbative, taking $m_{h_{2}}=300 \mathrm{GeV}$. In the purple region, the Higgs quartic coupling $\lambda_{H}$ is stabilized by the contributions of $\lambda_{H S}$ to its running under the renormalization. The red region can explain the cosmic ray anomalies consistently the constraints considered in the previous section. The other curves (solid green, dashed light blue, dot-dashed orange) represent the upper limit on $\lambda_{S}$ or $\sin \theta$ to avoid a Landau pole near the renormalization scale indicated. Bottom: Region that the scalar potential is stable and all the quartic couplings are perturbative up to the Planck scale (purple region) for $m_{h_{2}}=300,2000$, $10000 \mathrm{GeV}$. The red region is the same with the upper plot, but for $m_{h_{2}}=300 \mathrm{GeV}$ (solid) and $2000 \mathrm{GeV}$ (dashed).

$13 \mathrm{TeV}$ data at $m=95.3 \mathrm{GeV}$, with a production cross section times branching ratio of $0.13 \mathrm{pb}$, close to that which a SM Higgs of the same mass would produce [56,57]. To simultaneously accommodate both anomalies, we adopt the values taken in Ref. [85]

$$
\begin{gathered}
\frac{\sigma\left(e^{+} e^{-} \rightarrow h_{2} \rightarrow Z b \bar{b}\right)}{\sigma\left(e^{+} e^{-} \rightarrow h \rightarrow Z b \bar{b}\right)}=\mu_{\mathrm{LEP}}=0.117 \pm 0.057 \\
\frac{\sigma\left(g g \rightarrow h_{2} \rightarrow \gamma \gamma\right)}{\sigma(g g \rightarrow h \rightarrow \gamma \gamma)}=\mu_{\mathrm{CMS}}=0.6 \pm 0.2
\end{gathered}
$$

where $h$ denotes a SM-like Higgs with mass $96 \mathrm{GeV}$.
Although the LEP anomaly could be explained by Higgs mixing with angle $\sin \theta \sim \pm \sqrt{0.117}= \pm 0.34$, this would not be sufficient to produce the CMS excess. As pointed out in Ref. [60], one needs to couple $h_{2}$ to new charged states to enhance the branching ratio (by a factor of $\sim 7$ ) for $h_{2} \rightarrow \gamma \gamma$ via the one-loop diagram containing the exotic states.

\section{A. Adding charged scalars}

We investigated models with charged colored scalars $\Phi$ that decay into two or four quarks. One might think that the latter case is more difficult to probe at the LHC, since the signal would be pair production of $\Phi$ followed by decays into four jets each. However this mode has recently been tightly constrained by CMS [86], with $m_{\Phi}>710 \mathrm{GeV}$ $(1.4 \mathrm{TeV})$ for colored triplets (octets). It turns out that models where $\Phi$ can decay into two jets are less stringently constrained at present [87]. One could also consider models where $\Phi$ decays to six quarks through a dimension-10 operator. However we estimate that the scale $\Lambda$ suppressing such an operator must be $\lesssim 3 \mathrm{TeV}$ to avoid $\Phi$ exiting the detector before decaying, and being identified as a long-lived charged particle, which is also excluded for $m_{\Phi} \lesssim 1 \mathrm{TeV}$ for color triplets [88]. We do not consider this case in the following.

The models, which are summarized in Table I, are represented by the interaction Lagrangian

$$
\begin{aligned}
\mathcal{L} \ni & \lambda_{S \Phi}|S|^{2}|\Phi|^{2}+\lambda_{H \Phi}|H|^{2}|\Phi|^{2} \\
& +\left(y_{\Phi} \Phi^{*}\left(\bar{q}_{R} q_{R}^{c}\right) \operatorname{or} \frac{1}{\Lambda^{3}} \Phi\left(\bar{q}_{R} q_{R}^{c}\right)^{2}\right)+\text { H.c. }
\end{aligned}
$$

In the last term, a dimension-7 effective operator, the right-handed quarks are assumed to form a sextet or triplet of color, the smallest possible irreducible representations, which can combine with $\Phi$ to form a singlet. The possible flavor combinations are $q q q q=u и u и$, $u u u d$, uudd, uddd and $d d d d$ corresponding to charges $q_{\Phi}=8 / 3,5 / 3,2 / 3,-1 / 3$ and $-4 / 3$, respectively, where $u$ and $d$ refer to generic up- and down-type quark flavors. For the two-quark models, $\Phi$ could transform as a $\overline{6}$ or a 3 of $\mathrm{SU}(3)_{c}$, but we consider only the color triplet case, which is less strongly constrained by collider limits, with $q q=u u, u d, d d$ and corresponding charges $q_{\Phi}=4 / 3$, $1 / 3,-2 / 3$.

The first two interactions in (11) produce a shift in the mass of $\Phi$ when $S$ and $H$ get their VEVs. If $\mu_{\phi}^{2}$ denotes the original Lagrangian parameter, then the physical mass is given by

$$
\begin{aligned}
m_{\Phi}^{2} & =\mu_{\Phi}^{2}+\frac{1}{2} \lambda_{S \Phi} v_{s}^{2}+\frac{1}{2} \lambda_{H \Phi} v^{2} \\
& \equiv \mu_{\Phi}^{2}+\bar{\mu}_{\Phi}^{2} .
\end{aligned}
$$


TABLE I. Models of $\mathrm{SU}(3)_{c}$ sextet or triplet scalars coupling to $S$, considered for explaining the CMS diphoton excess. The mass $m_{\Phi}$ (in $\mathrm{GeV}$ ) and mixing angle are chosen to fit the combined LEP and CMS anomalies. $\bar{\mu}_{\Phi}$ represents the contribution of the $S$ and $H$ VEVs to the mass; see Eq. (12). The ratio of couplings $\lambda_{H \Phi} / \lambda_{S \Phi}$ is chosen to mitigate Higgs coupling strength deviations, leading to the reduced $\chi^{2}$ values in the last column. Models marked with $(*)$ can optionally refer to the two-quark coupling version, while those without asterisk couple $\Phi$ to four quarks. The magnitude of $\lambda_{S \Phi}$ is such that $m_{\Phi}$ saturates the experimental constraints on $\Phi$ decaying to $q q q q$ [86] (or $q q$ [89] for the models marked with “*”).

\begin{tabular}{lcccccccc}
\hline \hline Model & $q_{\Phi}$ & $N_{c}$ & $\frac{m_{\Phi}}{\left|\lambda_{S \Phi}\right|^{1 / 2}}$ & $\frac{\bar{\mu}_{\Phi}}{\left|\lambda_{S \Phi}\right|^{1 / 2}}$ & $s_{\theta}$ & $\lambda_{S \Phi}$ & $\lambda_{H \Phi}$ & $\chi^{2} /$ d.o.f. \\
\hline 1 & $8 / 3$ & 6 & 943 & 836 & 0.39 & 1.9 & 3.3 & 3.6 \\
2 & $8 / 3$ & 3 & 601 & 778 & 0.36 & 1.4 & 1.6 & 2.2 \\
3 & $5 / 3$ & 6 & 700 & 741 & 0.34 & 3.4 & 3.5 & 2.1 \\
4 & $5 / 3$ & 3 & 417 & 838 & 0.39 & 3.0 & 5.2 & 3.7 \\
5 & $2 / 3$ & 6 & 588 & 795 & 0.37 & 4.8 & 5.9 & 1.4 \\
$6(*)$ & $2 / 3$ & 3 & 284 & 765 & 0.35 & 3.4 & 3.6 & 1.5 \\
7 & $-1 / 3$ & 6 & 554 & 830 & 0.39 & 5.4 & 8.0 & 1.5 \\
$8(*)$ & $-1 / 3$ & 3 & 256 & 810 & 0.38 & 4.1 & 5.6 & 1.4 \\
9 & $-4 / 3$ & 6 & 666 & 752 & 0.35 & 3.8 & 3.9 & 1.8 \\
$10(*)$ & $-4 / 3$ & 3 & 333 & 737 & 0.34 & 2.4 & 3.0 & 2.5 \\
\hline \hline
\end{tabular}

One would like to avoid having $m_{\phi}^{2} \ll\left|\mu_{\phi}^{2}\right|$ or $\left|\bar{\mu}_{\phi}^{2}\right|$ since this would require fine-tuning; Table I shows that some mild tuning $(\sim 0.1)$ is needed for models 6 and 8 .

\section{B. Couplings to $g, \gamma, h_{1}$}

The effective couplings of $h_{1,2}$ to gluons and photons have been computed for a similar class of models [90],

$\mathcal{L}_{\text {eff }}=\sum_{i=1}^{2}\left(\frac{\alpha_{\mathrm{s}} b_{i}^{g}}{8 \pi v} h_{i} G_{\mu \nu}^{a} G^{a \mu \nu}+\frac{\alpha_{\mathrm{em}} b_{i}^{\gamma}}{8 \pi v} h_{i} F_{\mu \nu} F^{\mu \nu}\right)$

with

$$
\begin{aligned}
b_{1}^{\gamma} & =\frac{N_{c}}{3} q_{\Phi}^{2}\left(-s_{\theta} \eta_{S}+c_{\theta} \eta_{H}\right)+b_{\mathrm{SM}}^{\gamma} c_{\theta} \\
b_{1}^{g} & =R_{\Phi}\left(-s_{\theta} \eta_{S}+c_{\theta} \eta_{H}\right)+\frac{2}{3} c_{\theta} \\
b_{2}^{\gamma} & =\frac{N_{c}}{3} q_{\Phi}^{2}\left(c_{\theta} \eta_{S}+s_{\theta} \eta_{H}\right)+b_{\mathrm{SM}^{\prime} s_{\theta}}^{\gamma^{\prime}} \\
b_{2}^{g} & =R_{\Phi}\left(c_{\theta} \eta_{S}+s_{\theta} \eta_{H}\right)+\frac{2}{3} s_{\theta} .
\end{aligned}
$$

Here $N_{c}=6(3)$ is the number of colors of $\Phi, R_{\Phi}=$ $5 / 6(1 / 6)$ for sextet (triplet) scalars, $b_{\mathrm{SM}}^{\gamma} \cong-6.5$, $b_{\mathrm{SM}}^{\gamma^{\prime}} \cong-5.9,{ }^{2} \quad \eta_{S}=\lambda_{S \Phi} v v_{s} / m_{\Phi}^{2}$ and $\eta_{H}=\lambda_{H \Phi} v^{2} / m_{\Phi}^{2}$.

\footnotetext{
${ }^{2}$ This is somewhat smaller than the value for the SM Higgs, due to $m_{h_{2}}$ being less than $m_{h_{1}}$. $R_{\Phi}$ is equal to the index of the representation, divided by 6 for a complex scalar field.
}

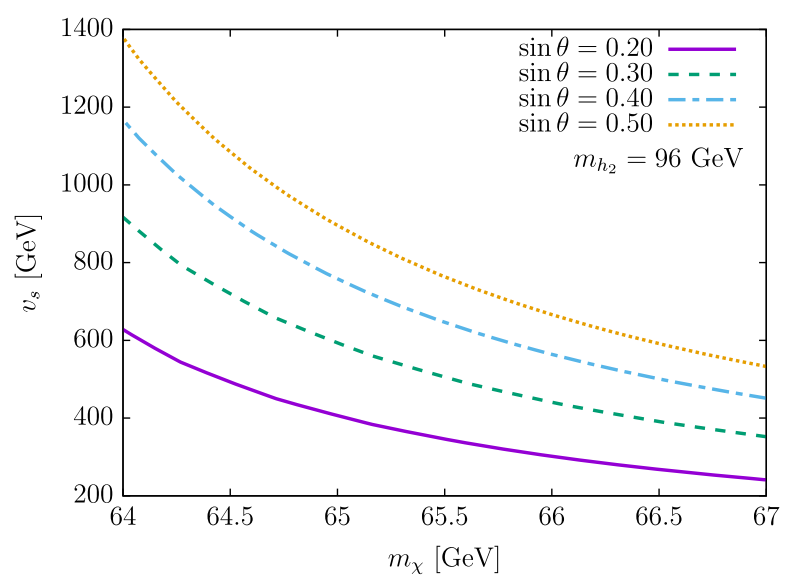

FIG. 4. The singlet VEV $v_{s}$ versus DM mass $m_{\chi}$ required by the relic abundance, for several choices of mixing angle, assuming $m_{h_{2}}=96 \mathrm{GeV}$.

The value of $v_{s}$ giving the correct relic density for $m_{h_{2}}=96 \mathrm{GeV}$ is plotted in Fig. 4 , and can be fit to $v_{s} \cong$ $\left(-35+3641 s_{\theta}-1603 s_{\theta}^{2}\right) \mathrm{GeV}$ at $m_{\chi}=64 \mathrm{GeV}$, while reaching smaller values $v_{s} \cong 250 \sim 550 \mathrm{GeV}$ at $m_{\chi}=$ $67 \mathrm{GeV}$. Since the solution for $m_{\Phi}$ derived below is proportional to $v_{s}^{1 / 2}$, in order to maximize the scale of new physics we adopt the lower value of $m_{\chi}$, which is consistent with the cosmic ray anomalies as described previously.

The couplings of the SM Higgs to gluons and photons get modified by mixing and the $\Phi$ loop. Couplings of $h_{1}$ to fermions and $W / Z$ bosons are reduced by the mixing effect alone. This can be quantified by $\kappa_{i}$ parameters, defined as the coupling to particle $i$ divided by the corresponding value in the SM:

$$
\kappa_{g}=\frac{b_{1}^{g}}{2 / 3}, \quad \kappa_{\gamma}=\frac{b_{1}^{\gamma}}{b_{\mathrm{SM}}^{\gamma}}, \quad \kappa_{W, Z}=\kappa_{f}=c_{\theta} .
$$

These factors appear in the signal strengths for various Higgs production and decay processes. In addition we make use of the ratio of branching ratios of the Higgs to different final states, relative to the corresponding SM values. If there was only mixing, these would be trivial, but because of the $\Phi$ loop, the $g g$ and $\gamma \gamma$ channels can change relative to the others. These factors are

$$
\begin{aligned}
c_{f f} & =\frac{1}{\operatorname{BR}(f)+\operatorname{BR}(g) \mu_{g} / c_{\theta}} \\
c_{\gamma \gamma} & =\frac{\mu_{\gamma}}{\operatorname{BR}(f) c_{\theta}^{2}+\operatorname{BR}(g) \mu_{g}}
\end{aligned}
$$

where $\operatorname{BR}(g)=0.086$ is the $\mathrm{SM}$ branching ratio into gluons at $m_{h}=96 \mathrm{GeV}$, and $\operatorname{BR}(f)=0.914$ is that into other states. Then for example the signal strength 
for $g g \rightarrow h \rightarrow \gamma \gamma$ is $\kappa_{g}^{2} c_{\gamma \gamma}$, while that for $g g \rightarrow h \rightarrow Z Z \rightarrow 4 \ell$ is $\kappa_{g}^{2} c_{f f}$.

\section{Fitting the anomalies} form

By taking the square root, Eq. (10) can be recast in the

$$
\begin{gathered}
s_{\theta}^{2}=\sqrt{\mu_{L \mathrm{EP}} \hat{\Gamma}_{h_{2}}} \\
\left(\frac{b_{2}^{g}}{2 / 3}\right)\left(\frac{b_{2}^{\gamma}}{b_{\mathrm{SM}}^{\gamma^{\prime}}}\right)= \pm \sqrt{\mu_{\mathrm{CMS}} \hat{\Gamma}_{h_{2}}}
\end{gathered}
$$

where $\hat{\Gamma}_{h_{2}}=\mathrm{BR}(f) s_{\theta}^{2}+\mathrm{BR}(g)\left(\frac{3}{2} b_{2}^{g}\right)^{2}$ is the ratio of the $h_{2}$ width to that of a SM-like Higgs of the same mass. For fixed values of $\lambda_{H \Phi}$ and $\lambda_{S \Phi}$, these can be solved numerically for $\theta$ and $m_{\Phi}$. The equations are only weakly coupled, through the small gluon contribution to $\hat{\Gamma}_{h_{2}}$; therefore $s_{\theta}$ is practically fixed by (17), while $m_{\Phi}$ is mostly determined by (18).

For solutions of (17) and (18), the Higgs couplings (15) depend upon the ratio $\eta_{H} / \eta_{S}=v \lambda_{H \Phi} /\left(v_{s} \lambda_{S \Phi}\right)$, which we determine by fitting to the set of signal strengths and effective couplings shown in Fig. 5. The $\chi^{2}$ per degree of freedom (five observations from combining ATLAS and CMS results, minus one parameter) is listed in Table I, and the results for $\kappa_{\gamma}$ versus $\kappa_{g}$ are illustrated in Fig. 5. There is a preference for models $5-8$, in which the scalar has charge $q_{\Phi}=2 / 3$ or $-1 / 3$, and can be either a triplet or a sextet. Models 2, 3 with $q_{\Phi}=8 / 3,5 / 3$ and $N_{c}=3,6$ respectively also give an acceptable fit, as well as model 9 with $q_{\Phi}=-4 / 3, N_{c}=6$.

The models face daunting constraints from LHC searches for pair production of colored scalars that decay into jets. Gluinos must be heavier than 1.5(1.4) $\mathrm{TeV}$ [86,87]

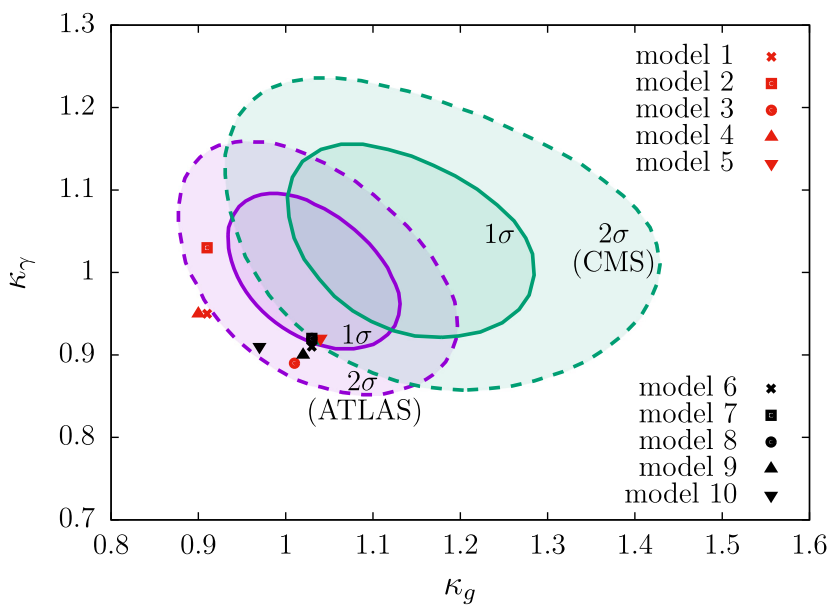

FIG. 5. Effective Higgs coupling strengths to photons versus gluons predicted by the 10 models, and the allowed regions from ATLAS [91] and CMS [92]. for decays into 2 (4) jets. ${ }^{3}$ Searches for $R$-parity violating top squark decays by CMS [89] and ATLAS [87,96-98] put complementary constraints on triplets decaying to two jets, that depend upon whether one of the jets is tagged for $b$ quarks: $m_{\tilde{t}}>520 \mathrm{GeV}$ for decays into first or second generation quarks $q q$, versus $m_{\tilde{t}}>610 \mathrm{GeV}$ for decays into $b q$.

Table II shows that one must take rather large values of the couplings $\lambda_{S \Phi}, \lambda_{H \Phi}$ to satisfy the collider limits on $m_{\Phi}$. Although these values are consistent with perturbative unitarity, they typically require some fine-tuning since the one-loop contributions to $\lambda_{H}, \lambda_{S}$ and $\lambda_{H S}$ are of order

$$
\delta \lambda_{H} \sim \frac{3 \lambda_{H \Phi}^{2}}{16 \pi^{2}}, \quad \delta \lambda_{H S} \sim \frac{3 \lambda_{H \Phi} \lambda_{S \Phi}}{16 \pi^{2}}, \quad \delta \lambda_{S} \sim \frac{3 \lambda_{S \Phi}^{2}}{16 \pi^{2}}
$$

which tend to be much larger than the tree-level values $\lambda_{H} \sim 0.24, \lambda_{S} \sim 0.009$ and $\lambda_{H S} \sim 0.008$. Model 2 however is relatively good in this respect, with $\delta \lambda_{H} \sim \delta \lambda_{H S} \sim$ $\delta \lambda_{S} \sim 0.04$, requiring only a mild 1 part in 5 tuning.

The $\Phi$-extended model needed for the collider anomalies, although not ruled out, is tightly constrained, and would likely require other new physics to be part of a UV-complete picture. For example, stability of the Higgs potential is not helped by the small $\lambda_{H S}$ coupling in these examples, although the much larger $\lambda_{H \Phi}$ coupling may do so. Pending experimental verification, we content ourselves with the foregoing low-energy description as concerns the tentative LEP/CMS excesses.

\section{Gamma-ray line searches}

The Fermi-LAT has obtained stringent constraints on DM annihilating to two monochromatic photons [99], which occurs through the Higgs portal in our model, and is potentially enhanced by the $\Phi$ induced coupling of photons to the singlet. The cross section for $\chi \chi \rightarrow \gamma \gamma$ by $h_{1,2}$ exchange in the $s$ channel is

$$
\left\langle\sigma_{\gamma \gamma} v_{\text {rel }}\right\rangle \approx \frac{\alpha_{\mathrm{em}}^{2} s}{128 \pi^{3} v^{2} v_{s}^{2}}\left|\frac{s_{\theta} m_{h_{1}}^{2} b_{1}^{\gamma}}{s-m_{h_{1}}^{2}}-\frac{c_{\theta} m_{h_{2}}^{2} b_{2}^{\gamma}}{s-m_{h_{2}}^{2}}\right|^{2}
$$

where $s=4 m_{\chi}^{2}$ and the decay width in the $s$-channel propagators were neglected. ${ }^{4}$ (Here we modify the definition of $b_{2}^{\gamma}$ to be in terms of $b_{\mathrm{SM}}^{\gamma}$ rather than $b_{\mathrm{SM}}^{\gamma^{\prime}}$ since the same energy $\sqrt{s}$ is flowing through the loops coupling to

\footnotetext{
${ }^{3}$ We have compared the leading-order production cross section for gluinos [93] to that of color sextet scalars [94] at the same mass and $\sqrt{s}=13 \mathrm{TeV}$, averaged over parton distribution functions [95], and found that they are quite similar, suggesting a bound of $1.3 \mathrm{TeV}$ for decays into four jets.

${ }^{4}$ There is also a contribution from direct coupling of $\chi \chi$ to $\gamma \gamma$ through the $\Phi$ loop, whose matrix element is $|\mathcal{M}|=$ $\alpha_{\mathrm{em}} q_{\Phi} N_{c} \lambda_{S \Phi} q_{\Phi}^{2} m_{\chi}^{2} /\left(3 \sqrt{2} \pi m_{\Phi}^{2}\right)$ [102], which is subdominant to the $h_{1,2}$-mediated contributions.
} 
TABLE II. Predicted SM Higgs signal strengths, and observed values, for the 10 models considered. Last column shows the cross section for $\chi \chi \rightarrow \gamma \gamma$ annihilation in the Galaxy, and at the bottom the limit [99] assuming Einasto profile for the Milky Way DM halo.

\begin{tabular}{|c|c|c|c|c|c|c|}
\hline Observable & $g g \rightarrow h_{1} \rightarrow \gamma \gamma \quad g$ & $g g \rightarrow h_{1} \rightarrow Z Z \rightarrow 4 \ell$ & $\kappa_{g}$ & $\kappa_{\gamma}$ & $\kappa_{Z, W}$ & $\left\langle\sigma_{\gamma \gamma} v_{\text {rel }}\right\rangle\left(10^{-28} \mathrm{~cm}^{3} / \mathrm{s}\right)$ \\
\hline Predicted & $\kappa_{g}^{2} c_{\gamma \gamma}$ & $\kappa_{g}^{2} c_{f f}$ & $\left|\frac{3}{2} b_{1}^{g}\right|$ & $\left|b_{1}^{\gamma} / b_{\mathrm{SM}}^{\gamma}\right|$ & $c_{\theta}$ & Eq. (20) \\
\hline Model 1 & 0.87 & 0.82 & 0.91 & 0.95 & 0.92 & 0.9 \\
\hline Model 2 & 1.02 & 0.83 & 0.91 & 1.03 & 0.93 & 1.1 \\
\hline Model 3 & 0.91 & 1.00 & 1.01 & 0.89 & 0.94 & 0.5 \\
\hline Model 4 & 0.87 & 0.82 & 0.90 & 0.95 & 0.92 & 0.9 \\
\hline Model 5 & 1.03 & 1.06 & 1.04 & 0.92 & 0.93 & 0.6 \\
\hline Model 6 & 0.99 & 1.04 & 1.03 & 0.91 & 0.94 & 0.6 \\
\hline Model 7 & 1.03 & 1.03 & 1.03 & 0.92 & 0.92 & 0.6 \\
\hline Model 8 & 1.02 & 1.04 & 1.03 & 0.92 & 0.93 & 0.6 \\
\hline Model 9 & 0.95 & 1.02 & 1.02 & 0.90 & 0.94 & 0.5 \\
\hline Model 10 & 0.88 & 0.94 & 0.97 & 0.91 & 0.94 & 0.5 \\
\hline CMS & $1.15 \pm 0.15[100]$ & $0.94 \pm 0.10$ & $1.18_{-0.14}^{+0.16}[92]$ & $1.07 \pm 0.15[92]$ & $\kappa_{Z}=1.00 \pm 0.11[92]$ & Fermi/LAT: \\
\hline ATLAS & $0.96 \pm 0.14[91]$ & $1.04_{-0.15}^{+0.16}[91]$ & $0.99_{-0.10}^{+0.11}[91]$ & $1.05 \pm 0.09[91]$ & $\begin{array}{c}\kappa_{W}=1.05 \pm 0.09 \\
\kappa_{Z}=1.11 \pm 0.08[91]\end{array}$ & $\lesssim(0.5-4)[99]$ \\
\hline Combined & $1.06 \pm 0.10$ & $0.99 \pm 0.10$ & $1.09_{-0.09}^{+0.10}$ & $1.06 \pm 0.09$ & $1.05 \pm 0.08$ & $<1.4$ \\
\hline
\end{tabular}

$h_{1}$ and $h_{2}$.) In the last column of Table II we show the predictions for the $\Phi$-extended models. The corresponding prediction for the minimal model with no scalar $\Phi$ can be obtained from this by setting $N_{c}=0$ in $b_{1,2}^{\gamma}$, and can always be made parametrically small by taking $s_{\theta} \ll 1$, unlike in the extended models. We find that the allowed regions shown in previous sections are not reduced by this constraint.

The gamma-ray line constraints on $\langle\sigma v\rangle_{\gamma \gamma}$ become stronger or weaker depending on how cuspy one assumes the density profile of DM is in the Milky Way. Therefore Ref. [99] considers four possibilities of varying cuspiness, resulting in a range of upper bounds shown in Table II. For reference we adopt an intermediate upper limit $\left\langle\sigma_{\gamma \gamma} v_{\text {rel }}\right\rangle<1.4 \times 10^{-28} \mathrm{~cm}^{3} / \mathrm{s}$ at $m_{\chi}=64 \mathrm{GeV}$, based on the assumption of an Einasto DM profile.

Interestingly the predicted values for $\langle\sigma v\rangle_{\gamma \gamma}$ are not far below the averaged upper limit, leading to the expectation that an observable gamma-ray line from the Galactic Center, of energy $\sim 64 \mathrm{GeV}$, should be correlated with the LEP and CMS anomalies. The predicted line will be tested by the next generation gamma-ray telescope GAMMA-400 [103].

\section{CONCLUSION}

Annihilation of $\mathcal{O}(60) \mathrm{GeV}$ mass dark matter into $b$ quarks remains as a plausible explanation for excess cosmic gamma rays and antiprotons that have been observed by Fermi/LAT and AMS respectively. We have shown that an economical model of scalar DM coupling to the Higgs can naturally explain these observations, while respecting other constraints. In particular, the pseudo-Goldstone nature of pNGB DM makes it immune to direct detection, because of its highly velocity-suppressed couplings to nucleons.

We showed that, while firmly within the preferred parameter space for antiprotons, our prediction for the DM mass $m_{\chi} \cong(64-67) \mathrm{GeV}$ is at the high end of the $2 \sigma$-allowed region for the Galactic Center gamma-ray excess, and the annihilation cross section is not far below the Fermi indirect detection limit from dwarf spheroidal galaxies. Further, if the extra $C P$-even Higgs boson is in the mass range $m_{h_{2}} \sim(200-600) \mathrm{GeV}$, this allowed region can overlap with parameters for which the Higgs potential remains stable up to the Planck scale, and the new quartic couplings are free from Landau poles.

By extending the model with an additional scalar $\Phi$ that carries charge and color, and assuming that $m_{h_{2}} \sim 96 \mathrm{GeV}$, we can also account for tentative excess $b$ quarks observed at LEP and diphotons at CMS. The most promising such model has $\Phi$ in the 3 representation, $m_{\Phi} \gtrsim 720 \mathrm{GeV}$, charge $q_{\Phi}=8 / 3$, and decaying into four up-type quarks in the first or second generations. It can eventually be ruled out by strengthened limits on pair production of such scalars, or by more precise observations of the production and decay modes of the standard model Higgs boson, with which it has some tensions at the $2 \sigma$ level. A further test is through the search for a monochromatic gamma-ray line at $\sim 64 \mathrm{GeV}$ from the Galactic Center, which could rule out the extended model with a modest increase in sensitivity over current limits. This extension would require some other new physics to stabilize the Higgs self-coupling and the new scalar cross-couplings at high scales. 
Higgs sectors extended by couplings to singlet fields have been invoked to strengthen the electroweak phase transition, possibly enabling electroweak baryogenesis or producing observable gravity waves. This is one feature that the pNGB DM does not have, however, as was shown in Ref. [104].

\section{ACKNOWLEDGMENTS}

This work was supported by the Natural Sciences and Engineering Research Council of Canada (NSERC), Compute Ontario, WestGrid, Compute Canada, and the Yukawa Institute Computer Facility.
[1] M. Ajello et al. (Fermi-LAT Collaboration), Fermi-LAT observations of high-energy $\gamma$-ray emission toward the galactic center, Astrophys. J. 819, 44 (2016).

[2] M. Ackermann et al. (Fermi-LAT Collaboration), The Fermi galactic center $\mathrm{GeV}$ excess and implications for dark matter, Astrophys. J. 840, 43 (2017).

[3] L. Goodenough and D. Hooper, Possible evidence for dark matter annihilation in the inner Milky Way from the Fermi gamma ray space telescope, arXiv:0910.2998.

[4] D. Hooper and L. Goodenough, Dark matter annihilation in the galactic center as seen by the Fermi gamma ray space telescope, Phys. Lett. B 697, 412 (2011).

[5] D. Hooper and T. Linden, On the origin of the gamma rays from the galactic center, Phys. Rev. D 84, 123005 (2011).

[6] D. Hooper and T. R. Slatyer, Two emission mechanisms in the Fermi bubbles: A possible signal of annihilating dark matter, Phys. Dark Universe 2, 118 (2013).

[7] T. Daylan, D. P. Finkbeiner, D. Hooper, T. Linden, S. K. N. Portillo, N. L. Rodd, and T. R. Slatyer, The characterization of the gamma-ray signal from the central Milky Way: A case for annihilating dark matter, Phys. Dark Universe 12, 1 (2016).

[8] F. Calore, I. Cholis, and C. Weniger, Background model systematics for the Fermi GeV excess, J. Cosmol. Astropart. Phys. 03 (2015) 038.

[9] K. N. Abazajian, N. Canac, S. Horiuchi, and M. Kaplinghat, Astrophysical and dark matter interpretations of extended gamma-ray emission from the galactic center, Phys. Rev. D 90, 023526 (2014).

[10] N. Mirabal, Dark matter vs. Pulsars: Catching the impostor, Mon. Not. R. Astron. Soc. 436, 2461 (2013).

[11] F. Calore, M. D. Mauro, and F. Donato, Diffuse gamma-ray emission from galactic pulsars, Astrophys. J. 796, 1 (2014).

[12] R. M. O'Leary, M. D. Kistler, M. Kerr, and J. Dexter, Young pulsars and the galactic center $\mathrm{GeV}$ gamma-ray excess, arXiv:1504.02477.

[13] R. T. Bartels, T. D. P. Edwards, and C. Weniger, Bayesian model comparison and analysis of the Galactic disc population of gamma-ray millisecond pulsars, Mon. Not. R. Astron. Soc. 481, 3966 (2018).

[14] T. D. Brandt and B. Kocsis, Disrupted globular clusters can explain the galactic center gamma ray excess, Astrophys. J. 812, 15 (2015).

[15] D. Hooper, I. Cholis, T. Linden, J. Siegal-Gaskins, and T. Slatyer, Pulsars cannot account for the inner galaxy's GeV excess, Phys. Rev. D 88, 083009 (2013).
[16] I. Cholis, D. Hooper, and T. Linden, Challenges in explaining the galactic center gamma-ray excess with millisecond pulsars, J. Cosmol. Astropart. Phys. 06 (2015) 043.

[17] D. Haggard, C. Heinke, D. Hooper, and T. Linden, Low mass X-ray binaries in the inner galaxy: Implications for millisecond pulsars and the GeV excess, J. Cosmol. Astropart. Phys. 05 (2017) 056.

[18] R. K. Leane and T. R. Slatyer, Dark matter strikes back at the galactic center, arXiv:1904.08430.

[19] R. Bartels, S. Krishnamurthy, and C. Weniger, Strong Support for the Millisecond Pulsar Origin of the Galactic Center GeV Excess, Phys. Rev. Lett. 116, 051102 (2016).

[20] S. K. Lee, M. Lisanti, B. R. Safdi, T. R. Slatyer, and W. Xue, Evidence for Unresolved $\gamma$-Ray Point Sources in the Inner Galaxy, Phys. Rev. Lett. 116, 051103 (2016).

[21] M. Aguilar et al. (AMS Collaboration), Antiproton Flux, Antiproton-to-Proton Flux Ratio, and Properties of Elementary Particle Fluxes in Primary Cosmic Rays Measured with the Alpha Magnetic Spectrometer on the International Space Station, Phys. Rev. Lett. 117, 091103 (2016).

[22] Y. Fujita, K. Kohri, R. Yamazaki, and K. Ioka, Is the PAMELA anomaly caused by supernova explosions near the Earth?, Phys. Rev. D 80, 063003 (2009).

[23] T. Bringmann, M. Vollmann, and C. Weniger, Updated cosmic-ray and radio constraints on light dark matter: Implications for the $\mathrm{GeV}$ gamma-ray excess at the Galactic center, Phys. Rev. D 90, 123001 (2014).

[24] M. Cirelli, D. Gaggero, G. Giesen, M. Taoso, and A. Urbano, Antiproton constraints on the $\mathrm{GeV}$ gamma-ray excess: A comprehensive analysis, J. Cosmol. Astropart. Phys. 12 (2014) 045.

[25] D. Hooper, T. Linden, and P. Mertsch, What does the PAMELA antiproton spectrum tell us about dark matter, J. Cosmol. Astropart. Phys. 03 (2015) 021.

[26] K. Kohri, K. Ioka, Y. Fujita, and R. Yamazaki, Can we explain AMS-02 antiproton and positron excesses simultaneously by nearby supernovae without pulsars or dark matter?, Prog. Theor. Exp. Phys. 2016, 021 E01 (2016).

[27] A. Cuoco, M. Krämer, and M. Korsmeier, Novel Dark Matter Constraints from Antiprotons in Light of AMS-02, Phys. Rev. Lett. 118, 191102 (2017).

[28] M. Y. Cui, Q. Yuan, Y. L. S. Tsai, and Y. Z. Fan, Possible Dark Matter Annihilation Signal in the AMS-02 Antiproton Data, Phys. Rev. Lett. 118, 191101 (2017).

[29] I. Cholis, T. Linden, and D. Hooper, A robust excess in the cosmic-ray antiproton spectrum: Implications for annihilating dark matter, Phys. Rev. D 99, 103026 (2019). 
[30] S. J. Lin, X. J. Bi, and P. F. Yin, Investigating the dark matter signal in the cosmic ray antiproton flux with the machine learning method, arXiv:1903.09545.

[31] M. Carena, J. Osborne, N. R. Shah, and C. E. M. Wagner, The return of the WIMP: Missing energy signals and the galactic center excess, arXiv:1905.03768.

[32] E. Aprile et al. (XENON Collaboration), Dark Matter Search Results from a One Ton-Year Exposure of XENON1T, Phys. Rev. Lett. 121, 111302 (2018).

[33] J. A. Casas, D. G. Cerdeño, J. M. Moreno, and J. Quilis, Reopening the Higgs portal for single scalar dark matter, J. High Energy Phys. 05 (2017) 036.

[34] J. M. Cline, K. Kainulainen, P. Scott, and C. Weniger, Update on scalar singlet dark matter, Phys. Rev. D 88, 055025 (2013); Erratum, Phys. Rev. D92, 039906(E) (2015).

[35] P. Athron et al. (GAMBIT Collaboration), Status of the scalar singlet dark matter model, Eur. Phys. J. C 77, 568 (2017).

[36] S. Ipek, D. McKeen, and A. E. Nelson, A renormalizable model for the galactic center gamma ray excess from dark matter annihilation, Phys. Rev. D 90, 055021 (2014).

[37] M. Escudero, D. Hooper, and S. J. Witte, Updated collider and direct detection constraints on dark matter models for the galactic center gamma-ray excess, J. Cosmol. Astropart. Phys. 02 (2017) 038.

[38] T. Abe, M. Fujiwara, and J. Hisano, Loop corrections to dark matter direct detection in a pseudoscalar mediator dark matter model, J. High Energy Phys. 02 (2019) 028.

[39] C. Boehm, M. J. Dolan, C. McCabe, M. Spannowsky, and C. J. Wallace, Extended gamma-ray emission from coy dark matter, J. Cosmol. Astropart. Phys. 05 (2014) 009.

[40] C. Arina, E. Del Nobile, and P. Panci, Dark Matter with Pseudoscalar-Mediated Interactions Explains the DAMA Signal and the Galactic Center Excess, Phys. Rev. Lett. 114, 011301 (2015).

[41] V. Barger, P. Langacker, M. McCaskey, M. RamseyMusolf, and G. Shaughnessy, Complex singlet extension of the Standard Model, Phys. Rev. D 79, 015018 (2009).

[42] V. Barger, M. McCaskey, and G. Shaughnessy, Complex scalar dark matter vis-à-vis CoGeNT, DAMA/LIBRA and XENON100, Phys. Rev. D 82, 035019 (2010).

[43] C. Gross, O. Lebedev, and T. Toma, Cancellation Mechanism for Dark-Matter-Nucleon Interaction, Phys. Rev. Lett. 119, 191801 (2017).

[44] D. Azevedo, M. Duch, B. Grzadkowski, D. Huang, M. Iglicki, and R. Santos, One-loop contribution to darkmatter-nucleon scattering in the pseudoscalar dark matter model, J. High Energy Phys. 01 (2019) 138.

[45] K. Ishiwata and T. Toma, Probing pseudo NambuGoldstone boson dark matter at loop level, J. High Energy Phys. 12 (2018) 089.

[46] K. Huitu, N. Koivunen, O. Lebedev, S. Mondal, and T. Toma, Probing pseudo-Goldstone dark matter at the LHC, Phys. Rev. D 100, 015009 (2019).

[47] D. Karamitros, Pseudo Nambu-Goldstone dark matter: Examples of vanishing direct detection cross section, Phys. Rev. D 99, 095036 (2019).

[48] T. Alanne, M. Heikinheimo, V. Keus, N. Koivunen, and K. Tuominen, Direct and indirect probes of Goldstone dark matter, Phys. Rev. D 99, 075028 (2019).
[49] G. Degrassi, S. Di Vita, J. Elias-Miro, J. R. Espinosa, G. F. Giudice, G. Isidori, and A. Strumia, Higgs mass and vacuum stability in the Standard Model at NNLO, J. High Energy Phys. 08 (2012) 098.

[50] O. Lebedev, On stability of the electroweak vacuum and the Higgs portal, Eur. Phys. J. C 72, 2058 (2012).

[51] J. Elias-Miro, J. R. Espinosa, G. F. Giudice, H. M. Lee, and A. Strumia, Stabilization of the electroweak vacuum by a scalar threshold effect, J. High Energy Phys. 06 (2012) 031.

[52] V. V. Khoze, C. McCabe, and G. Ro, Higgs vacuum stability from the dark matter portal, J. High Energy Phys. 08 (2014) 026.

[53] A. Falkowski, C. Gross, and O. Lebedev, A second Higgs from the Higgs portal, J. High Energy Phys. 05 (2015) 057.

[54] P. Athron, J. M. Cornell, F. Kahlhoefer, J. Mckay, P. Scott, and S. Wild, Impact of vacuum stability, perturbativity and XENON1T on global fits of $\mathbb{Z}_{2}$ and $\mathbb{Z}_{3}$ scalar singlet dark matter, Eur. Phys. J. C 78, 830 (2018).

[55] R. Barate et al. (ALEPH, DELPHI, L3, and OPAL Collaborations and LEP Working Group), Search for the Standard Model Higgs boson at LEP, Phys. Lett. B 565, 61 (2003).

[56] CMS Collaboration, Search for new resonances in the diphoton final state in the mass range between 80 and $115 \mathrm{GeV}$ in pp collisions at $\sqrt{s}=8 \mathrm{TeV}$, Report No. CMS-PAS-HIG-14-037.

[57] CMS Collaboration, Search for new resonances in the diphoton final state in the mass range between 70 and $110 \mathrm{GeV}$ in pp collisions at $\sqrt{s}=8$ and $13 \mathrm{TeV}$, Report No. CMS-PAS-HIG-17-013.

[58] A. M. Sirunyan et al. (CMS Collaboration), Search for a Standard Model-like Higgs boson in the mass range between 70 and $110 \mathrm{GeV}$ in the diphoton final state in proton-proton collisions at $\sqrt{s}=8$ and $13 \mathrm{TeV}$, Phys. Lett. B 793, 320 (2019).

[59] J. Cao, X. Guo, Y. He, P. Wu, and Y. Zhang, Diphoton signal of the light Higgs boson in natural NMSSM, Phys. Rev. D 95, 116001 (2017).

[60] P. J. Fox and N. Weiner, Light signals from a lighter Higgs, J. High Energy Phys. 08 (2018) 025.

[61] U. Haisch and A. Malinauskas, Let there be light from a second light Higgs doublet, J. High Energy Phys. 03 (2018) 135.

[62] D. Liu, J. Liu, C. E. M. Wagner, and X. P. Wang, A light Higgs at the LHC and the B-anomalies, J. High Energy Phys. 06 (2018) 150.

[63] R. Vega, R. Vega-Morales, and K. Xie, Light (and darkness) from a light hidden Higgs, J. High Energy Phys. 06 (2018) 137.

[64] L. Liu, H. Qiao, K. Wang, and J. Zhu, A light scalar in the minimal dilaton model in light of LHC constraints, Chin. Phys. C 43, 023104 (2019).

[65] T. Biekötter, S. Heinemeyer, and C. Muñoz, Precise prediction for the Higgs-boson masses in the $\mu \nu$ SSM, Eur. Phys. J. C 78, 504 (2018).

[66] F. Domingo, S. Heinemeyer, S. Paßehr, and G. Weiglein, Decays of the neutral Higgs bosons into SM fermions and gauge bosons in the $\mathcal{C} \mathcal{P}$-violating NMSSM, Eur. Phys. J. C 78, 942 (2018). 
[67] T. Biekötter, M. Chakraborti, and S. Heinemeyer, A $96 \mathrm{GeV}$ Higgs Boson in the N2HDM, arXiv:1903.11661.

[68] T. Biekötter, M. Chakraborti, and S. Heinemeyer, An N2HDM solution for the possible $96 \mathrm{GeV}$ excess, arXiv: 1905.03280.

[69] G. Bélanger, F. Boudjema, A. Goudelis, A. Pukhov, and B. Zaldivar, Micromegas5.0: Freeze-in, Comput. Phys. Commun. 231, 173 (2018).

[70] M. Ibe, H. Murayama, and T. T. Yanagida, Breit-Wigner enhancement of dark matter annihilation, Phys. Rev. D 79, 095009 (2009).

[71] M. Ackermann et al. (Fermi-LAT Collaboration), Searching for Dark Matter Annihilation from Milky Way dwarf Spheroidal Galaxies with Six Years of Fermi Large Area Telescope Data, Phys. Rev. Lett. 115, 231301 (2015).

[72] A. Drlica-Wagner et al. (Fermi-LAT and DES Collaborations), Search for gamma-ray emission from DES dwarf spheroidal galaxy candidates with Fermi-LAT data, Astrophys. J. 809, L4 (2015).

[73] M. L. Ahnen et al. (MAGIC and Fermi-LAT Collaborations), Limits to dark matter annihilation cross-section from a combined analysis of MAGIC and Fermi-LAT observations of dwarf satellite galaxies, J. Cosmol. Astropart. Phys. 02 (2016) 039.

[74] A. M. Sirunyan et al. (CMS Collaboration), Search for invisible decays of a Higgs boson produced through vector boson fusion in proton-proton collisions at $\sqrt{s}=13 \mathrm{TeV}$, Phys. Lett. B 793, 520 (2019).

[75] M. Aaboud et al. (ATLAS Collaboration), Combination of Searches for Invisible Higgs Boson Decays with the ATLAS Experiment, Phys. Rev. Lett. 122, 231801 (2019).

[76] S. Ando and K. Ishiwata, Constraining particle dark matter using local galaxy distribution, J. Cosmol. Astropart. Phys. 06 (2016) 045.

[77] A. Albert et al. (Fermi-LAT and DES Collaborations), Searching for dark matter annihilation in recently discovered Milky Way satellites with Fermi-LAT, Astrophys. J. 834, 110 (2017).

[78] C. Y. Chen, S. Dawson, and I. M. Lewis, Exploring resonant di-Higgs boson production in the Higgs singlet model, Phys. Rev. D 91, 035015 (2015).

[79] T. Binder, T. Bringmann, M. Gustafsson, and A. Hryczuk, Early kinetic decoupling of dark matter: When the standard way of calculating the thermal relic density fails, Phys. Rev. D 96, 115010 (2017).

[80] M. Duch and B. Grzadkowski, Resonance enhancement of dark matter interactions: the case for early kinetic decoupling and velocity dependent resonance width, J. High Energy Phys. 09 (2017) 159.

[81] A. Hektor, A. Hryczuk, and K. Kannike, Improved bounds on $\mathbb{Z}_{3}$ singlet dark matter, J. High Energy Phys. 03 (2019) 204.

[82] W. E. East, J. Kearney, B. Shakya, H. Yoo, and K. M. Zurek, Spacetime dynamics of a Higgs vacuum instability during inflation, Phys. Rev. D 95, 023526 (2017).

[83] F. Staub, Automatic calculation of supersymmetric renormalization group equations and self energies, Comput. Phys. Commun. 182, 808 (2011).

[84] F. Staub, SARAH 4: A tool for (not only SUSY) model builders, Comput. Phys. Commun. 185, 1773 (2014).
[85] S. Heinemeyer and T. Stefaniak, A Higgs boson at $96 \mathrm{GeV}$, Proc. Sci., CHARGED2018 (2019) 016.

[86] A. M. Sirunyan et al. (CMS Collaboration), Search for Pair-Produced Resonances Each Decaying into at Least Four Quarks in Proton-Proton Collisions at $\sqrt{s}=13 \mathrm{TeV}$, Phys. Rev. Lett. 121, 141802 (2018).

[87] M. Aaboud et al. (ATLAS Collaboration), A search for pair-produced resonances in four-jet final states at $\sqrt{s}=13 \mathrm{TeV}$ with the ATLAS detector, Eur. Phys. J. C 78, 250 (2018).

[88] V. Khachatryan et al. (CMS Collaboration), Search for long-lived charged particles in proton-proton collisions at $\sqrt{s}=13$ TeV, Phys. Rev. D 94, 112004 (2016).

[89] A. M. Sirunyan et al. (CMS Collaboration), Search for pair-produced resonances decaying to quark pairs in proton-proton collisions at $\sqrt{s}=13 \mathrm{TeV}$, Phys. Rev. D 98, 112014 (2018).

[90] K. Nakamura, K. Nishiwaki, K. y. Oda, S. C. Park, and Y. Yamamoto, Di-Higgs enhancement by neutral scalar as probe of new colored sector, Eur. Phys. J. C 77, 273 (2017).

[91] ATLAS Collaboration, Combined measurements of Higgs boson production and decay using up to $80 \mathrm{fb}^{-1}$ of protonproton collision data at $\sqrt{s}=13 \mathrm{TeV}$ collected with the ATLAS experiment, Report No. ATLAS-CONF-2019-005.

[92] A. M. Sirunyan et al. (CMS Collaboration), Combined measurements of Higgs boson couplings in proton-proton collisions at $\sqrt{s}=13 \mathrm{TeV}$, Eur. Phys. J. C 79, 421 (2019).

[93] W. Beenakker, R. Hopker, M. Spira, and P. M. Zerwas, Gluino pair production at the Tevatron, Z. Phys. C 69, 163 (1995).

[94] C. R. Chen, W. Klemm, V. Rentala, and K. Wang, Color sextet scalars at the CERN large hadron collider, Phys. Rev. D 79, 054002 (2009).

[95] A. D. Martin, W. J. Stirling, R. S. Thorne, and G. Watt, Parton distributions for the LHC, Eur. Phys. J. C 63, 189 (2009).

[96] V. Khachatryan et al. (CMS Collaboration), Search for pair-produced resonances decaying to jet pairs in protonproton collisions at $\sqrt{s}=8 \mathrm{TeV}$, Phys. Lett. B 747, 98 (2015).

[97] V. Veeraraghavan (ATLAS Collaboration), A search for R-parity violating decays of the top squark in four-jet final states with the ATLAS experiment at $\sqrt{s}=13 \mathrm{TeV}$, Proc. Sci., LHCP2016 (2016) 235.

[98] ATLAS Collaboration, Search for supersymmetry in events with $b$-tagged jets and missing transverse energy in $p p$ collisions at $\sqrt{s}=13 \mathrm{TeV}$ with the ATLAS detector, Report No. ATLAS-CONF-2017-038.

[99] M. Ackermann et al. (Fermi-LAT Collaboration), Updated search for spectral lines from Galactic dark matter interactions with pass 8 data from the Fermi Large Area Telescope, Phys. Rev. D 91, 122002 (2015).

[100] CMS Collaboration, Measurements of Higgs boson production via gluon fusion and vector boson fusion in the diphoton decay channel at $\sqrt{s}=13 \mathrm{TeV}$, Report No. CMS-PAS-HIG-18-029.

[101] CMS Collaboration, Measurements of properties of the Higgs boson in the four-lepton final state in proton-proton 
collisions at $\sqrt{s}=13 \mathrm{TeV}$, Report No. CMS-PAS-HIG19-001.

[102] J. M. Cline, $130 \mathrm{GeV}$ dark matter and the Fermi gammaray line, Phys. Rev. D 86, 015016 (2012).

[103] A. M. Galper et al., Precision measurements of high-energy cosmic gamma-ray emission with the GAMMA-400 gamma-ray telescope, Yad. Fiz. 80, 660 (2017) [Phys. At. Nucl. 80, 1141 (2017)].

[104] K. Kannike and M. Raidal, Phase transitions and gravitational wave tests of pseudo-goldstone dark matter in the softly broken U(1) scalar singlet model, Phys. Rev. D 99, 115010 (2019). 